\title{
Visual and auditory accessory stimulus offset and the Simon effect
}

\author{
Akio Nishimura and Kazuhiko Yokosawa \\ University of Tokyo, Tokyo, Japan
}

\begin{abstract}
We investigated the effect on the right and left responses of the disappearance of a task-irrelevant stimulus located on the right or left side. Participants pressed a right or left response key on the basis of the color of a centrally located visual target. Visual (Experiment 1) or auditory (Experiment 2) task-irrelevant accessory stimuli appeared or disappeared at locations to the right or left of the central target. In Experiment 1, responses were faster when onset or offset of the visual accessory stimulus was spatially congruent with the response. In Experiment 2, responses were again faster when onset of the auditory accessory stimulus and the response were on the same side. However, responses were slightly slower when offset of the auditory accessory stimulus and the response were on the same side than when they were on opposite sides. These findings indicate that transient change information is crucial for a visual Simon effect, whereas sustained stimulation from an ongoing stimulus also contributes to an auditory Simon effect.
\end{abstract}

Perceptual events affect our actions. For example, performance is better when a spatial stimulus is assigned to an ipsilateral response as opposed to a contralateral response. This is known as the spatial stimulus-response compatibility (SRC) effect (Kornblum, Hasbroucq, \& Osman, 1990; Proctor \& Vu, 2006; Umiltà \& Nicoletti, 1990). The spatial relationship between a stimulus and a requisite response affects performance even when stimulus location is irrelevant to the task. Responses to a nonspatial property (e.g., color) of a target stimulus are faster and more accurate when the responding action (e.g., a buttonpress) occurs on a side that corresponds to the location of a target presentation than when the response occurs on a side opposite to the target's location (the Simon effect) (Kornblum et al., 1990; Lu \& Proctor, 1995; Simon, 1990; Simon \& Small, 1969).

The Simon effect emerges not only when spatial information is associated with a target stimulus, but also when it is associated with a visual or auditory accessory stimulus namely, stimuli that occur with a target but that are irrelevant to the task (see, e.g., Moore, Lleras, Grosjean, \& Marrara, 2004; Proctor, Pick, Vu, \& Anderson, 2005; Simon \& Pouraghabagher, 1978; Treccani, Umiltà, \& Tagliabue, 2006). This is termed the accessory stimulus Simon effect, or simply the accessory Simon effect. In a typical visual accessory Simon task, participants press a right or left key to identify, respectively, different colors (or shapes, etc.) of a centrally presented target. Simultaneously with a target presentation, a task-irrelevant visual stimulus is presented to either the right or the left of the central target. In a typical auditory accessory Simon task, a task-irrelevant tone is presented to the right or left ear simultaneously with the presentation of a central target. In both of these accessory Simon tasks, performance is better when a task-irrelevant stimulus occurs on the same side as a response than when it occurs on the opposite side. The accessory Simon effect indicates that presentation of a stimulus automatically activates the spatially congruent response, regardless of the task relevancy of the stimulus.

In previous studies that have shown effects of spatial stimulus presentations, two constituent factors may have been responsible for the observed phenomenon. One factor concerns the mere existence of a visual or auditory object as a source of stimulation at that particular spatial location. The other is a transient change at that location. In the present study, we dissociated these two factors by using the disappearance of an accessory stimulus as a basis for change. With the appearance of a stimulus, an object as a source of persisting stimulation is on the same side as the side where change occurs. However, with the disappearance of a stimulus, on either the right or the left side, the remaining object necessarily persists; therefore, it could function as a critical source of ongoing stimulation located on the side opposite to the side of a change. Our manipulation allowed us to differentiate the persistence of a stimulus at a given location from a change at that location and thereby to ascertain which type of perceptual information automatically activates a spatially compatible response. Not only does the sudden appearance of a stimulus attract attention (e.g., Posner, 1980), but so does the disappearance of a stimulus (e.g., Chastain \& Cheal, 2001; Theeuwes, 1991). This suggests the importance for our cognitive system of change information that accompanies both the appearance and disappearance of stimuli in spatial processing.

K. Yokosawa, yokosawa@L.u-tokyo.ac.jp 
Simon, Craft, and Webster (1971) investigated the effect of stimulus offset on response selection using visual (Experiment 1) and auditory (Experiment 2) SRC tasks. In their Experiment 1, participants pressed one of two response buttons with their right index fingers. At the beginning of each trial, both response buttons were lighted, but subsequently one button was extinguished. In one trial block, participants had to press the button that turned off, whereas in another block they had to press the button that remained lit. Performance was better when participants pressed the button that turned off than when they pressed the button that remained lit. This finding suggests the importance of change information in this task. In their Experiment 2 , one of two identical tones - initially presented to the right and left ears, respectively - was extinguished. Participants pressed either the right or the left key with their corresponding index fingers. In this case, and in contrast to the findings of Experiment 1, performance was better when participants pressed the response key opposite to the side on which the tone was terminated.

In short, in the effect of perception on action, it appears that when stimulus location is relevant to a task, and hence participants must intentionally process the spatial information of a stimulus, transient change information is important for the visual modality, whereas the sustained stimulation is important for the auditory modality. However, factors other than the modality itself might be responsible for this differential impact of visual versus auditory stimulation in Simon et al. (1971). In Simon et al. (1971), visual stimuli (lights) were presented directly on the response buttons in Experiment 1: The visual stimulus offset attracted attention, and participants had to reach toward and press the target button. Therefore, their findings may have reflected the advantage of a unitary target for visual attention and for goal-directed action (Deubel, Schneider, \& Paprotta, 1998). However, this was not the case for their Experiment 2. In this experiment, identical auditory stimuli (tones) were presented to the right and left ears and participants pressed right or left keys that differed from the locus of the source for these sound stimuli. Moreover, as Simon et al. (1971) pointed out, the abrupt change from a binaural tone to a monaural tone led to perception of a single tone's onset in the ear where the remaining tone continued to sound rather than tone offset in the opposite ear, where a tone ceased to be audible.

Using the Simon effect, Wühr and Kunde (2006) investigated the effect of visual stimulus disappearance when the spatial location of a stimulus was task irrelevant. In each trial, four stimuli were simultaneously presented at top right, bottom right, bottom left, and top left: one green and one red on the right side, and one green and one red on the left side. On a given trial, one of these stimuli disappeared while the others remained on (Experiment 3 ) or were masked (Experiment 4). Participants made a right or left response on the basis of the color of the stimulus that disappeared. In these experiments, an offset Simon effect was obtained in which performance was better when the response and the stimulus disappearance were on the same side than when they were on opposite sides. However, this result could be explained not only by the stimulus offset
Simon effect but also by a memory-based Simon effect. That is, when participants respond on the basis of the nonspatial feature of a memorized stimulus (which is no longer present), the remembered stimulus location affects responding (Hommel, 2002; Zhang \& Johnson, 2004). Thus, retrieving the color of a stimulus can automatically activate its spatial locus, thereby inducing a memory-based Simon effect. In Wühr and Kunde, a stimulus turned out to be the target only after it had disappeared. As a consequence, participants had to retrieve the color of the target stimulus. Therefore, the results of Wühr and Kunde may not have resulted from the offset of visual information itself but instead may have resulted from an automatic retrieval/ activation of a spatial code related to the target stimulus, which accompanies retrieval of a target's color.

In the present study, we sought to determine information that can automatically activate an ipsilateral response in the Simon effect. We introduced a stimulus offset manipulation and compared results in this condition with results in a stimulus onset condition using stimuli involving both visual and auditory modalities. In the stimulus onset condition, a transient change provided by stimulus appearance and an object as a source of persisting stimulation were on the same side. In the stimulus offset condition, a transient change provided by stimulus disappearance and the remaining object that persisted to function as a source of ongoing stimulation were on opposite sides. In order to eliminate the possibility of a memory-based effect and to isolate the effect of offset information, we used the accessory version of the Simon task. In an offset change condition, participants pressed the right or left key for the color of a visual target presented at the center of the display; the target was flanked by two accessory stimuli that occurred simultaneously with presentation of the target's color. One of the two accessory stimuli, on either the right or the left of the target, disappeared, while the other accessory stimulus remained on. Thus, in the present study, the task-relevant information signaling the correct response (i.e., target color) was presented directly on the target display; in other words, target information could be obtained without reliance on memory (i.e., online) during selection and execution of the response. In addition, the accessory stimuli did not need to be retrieved because they were, by definition, irrelevant; only the target stimulus carried task-relevant (color) information. This design enabled us to selectively test the effect of pure offset information in the visual or auditory modality.

On the basis of findings with visual stimulus offset reported in previous studies (Simon et al., 1971; Wühr \& Kunde, 2006), we predicted that the change information would be crucial in the Simon effect. To assess this, we defined a compatibility variable on the basis of the spatial correspondence between the response and the side of an accessory stimulus change (i.e., stimulus appearance in the onset condition and disappearance in the offset condition). If, as we predict, perceptual change information automatically activates the feature-sharing response, then the Simon effect would be comparable in size for the onset and offset conditions. If, on the other hand, the perceptual information that induces the Simon effect is an ongoing 
(i.e., persisting) stimulation due to the continued presence of a stimulus in a particular location, then the reversed Simon effect would be obtained for the offset condition, and it would be comparable in size to the Simon effect for the onset condition. Finally, if both perceptual change and ongoing stimulation from a stimulus contribute to the Simon effect, then the size of the Simon effect for the offset condition, regardless of whether it is positive or negative, would be smaller than the size of the Simon effect for the onset condition. We used visual accessory stimuli in Experiment 1 and auditory accessory stimuli in Experiment 2 to investigate possible differences between these modalities in information processing.

\section{EXPERIMENT 1 Visual Accessory Stimulus}

In Experiment 1, we used visual accessory stimuli that were irrelevant to the task. Each trial in the offset condition began with the presentation of a central fixation cross, together with right and left accessory stimuli. Simultaneously with a color change of the fixation stimulus, either the right or the left accessory stimulus disappeared, while the other accessory stimulus remained unchanged. Participants were told to press the right or left key, respectively, to indicate the relevant color change of the centrally located cross. We compared the results in this offset condition with results from an onset condition in which a single accessory stimulus appeared on the right or left of the fixation simultaneously with the color change of the fixation stimulus. The accessory stimulus change condition (onset, offset) was a between-participants factor in Experiment 1A and a within-participants factor in Experiment 1B.

\section{Experiment 1A: Condition As a Between-Participants Factor}

\section{Method}

Participants. Thirty-two graduate and undergraduate students (14 females; 2 left-handed), $20-24$ years of age $(M=21.3)$, participated in this experiment. All of them reported that they had normal or corrected-to-normal vision.

Apparatus and Stimuli. Stimulus presentation and data acquisition were controlled by an AV tachistoscope system (Iwatsu ISEL IS-703). Stimuli were presented on a 22 -in. display. The viewing distance (ca. $57 \mathrm{~cm}$ ) was maintained by a head- and chinrest. A response was accomplished by a keypress to one of two (left, right) response buttons separated by $7 \mathrm{~cm}$ and arranged approximately $21 \mathrm{~cm}$ in front of the participants.

The visual stimuli involved a central cross $(5 \times 5 \mathrm{~mm})$ and two 9 -mm squares to the right and left of the central cross. The fixation cross and a given square were separated by $36 \mathrm{~mm}$ (center to center). They were presented on a black background.

Task and Procedure. Participants were tested individually in a darkened room. Participants were randomly assigned to either the onset condition or the offset condition. For the onset condition, each trial started with a 2,000-msec presentation of a white fixation cross at the center of the display. The fixation cross then changed color, to either green or red. Simultaneously with the color change of the fixation, a white square (i.e., an accessory visual stimulus) appeared to the right or left of the central cross. After 2,000 msec, all the stimuli disappeared and the screen went blank. The intertrial interval was $1,000 \mathrm{msec}$. For the offset condition, each trial started with simultaneous presentation $(2,000 \mathrm{msec})$ of a white central fixation cross and two white squares (i.e., visual accessory stimuli) to the right and left of the fixation cross. The fixation cross then changed to green or red. Simultaneously with the color change of the fixation, one of the accessory stimuli (i.e., the right and left squares) disappeared, while the other remained on the screen. After 2,000 msec, all the remaining stimuli disappeared and the screen went blank. Again, the intertrial interval was $1,000 \mathrm{msec}$.

The participants' task was to press the right or left key as quickly and accurately as possible according to the color (green or red) of the central cross in both the onset and the offset conditions. Half the participants in each condition pressed the right key with their right index fingers in response to the red stimulus and the left key with their left index fingers in response to the green stimulus. The colorto-key mapping was reversed for the remaining half. Thus, the right and left squares were task-irrelevant, accessory visual stimuli.

The compatibility manipulation was based on the spatial correspondence between a response and the side on which an irrelevant accessory stimulus appeared (the onset condition) or disappeared (the offset condition). On half of the trials, the designated response involved a response key on the same side as a change of an accessory stimulus (the compatible condition); on the remaining trials, the response and accessory stimulus change were scheduled for opposite sides (the incompatible condition).

A session consisted of three test blocks ( 80 trials per block), preceded by a practice block of 16 trials. Participants could take short rests between the blocks.

\section{Results}

Trials on which participants failed to respond or on which reaction times (RTs) were shorter than $200 \mathrm{msec}$ or longer than $1,000 \mathrm{msec}$ were excluded from the data analyses $(0.55 \%$ of all the trials). Mean RTs for correct responses and the percentages of errors were submitted to separate two-factor ANOVAs with condition (onset, offset) as a between-participants factor and compatibility (compatible, incompatible) as a within-participants factor. Results are shown in Figure 1.

RT. The main effect of compatibility was significant $\left[F(1,30)=55.00, p<.001, \eta_{\mathrm{p}}^{2}=.647\right]$, indicating the Simon effect. Overall, responses were faster for the compatible condition $(M=391 \mathrm{msec})$ than for the incompatible condition $(M=413 \mathrm{msec})$. The two-way interaction between condition and compatibility was not significant $(F<1)$. The Simon effect was comparable in size between the onset condition $(19 \mathrm{msec})$ and the offset condition $(25 \mathrm{msec})$. Finally, the main effect of condition was not significant $(p>.29)$.

Error rate. The main effect of compatibility was significant $\left[F(1,30)=20.06, p<.001, \eta_{\mathrm{p}}^{2}=.401\right]$. Responses were more accurate for the compatible condition (1.2\%) than for the incompatible condition (3.3\%). Neither the main effect of condition nor the interaction between condition and compatibility was significant $(F \mathrm{~s}<1)$. The Simon effect was $1.7 \%$ for the onset condition and $2.5 \%$ for the offset condition.

\section{Experiment 1B: Condition As a Within-Participants Factor}

To assess whether the null effect of the change variable (onset vs. offset) was due to the fact that this condition reflected a between-participants manipulation, we replicated Experiment $1 \mathrm{~A}$ with the change condition manipulated as a within-participants and within-block variable. 


\section{Experiment 1A}

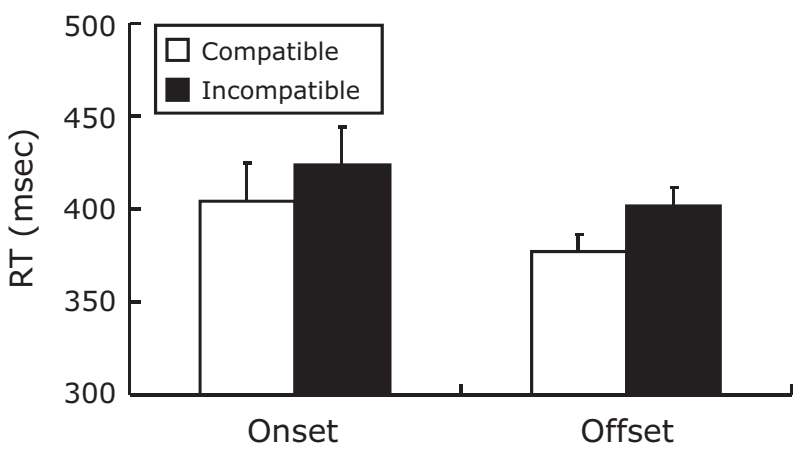

Experiment 1B

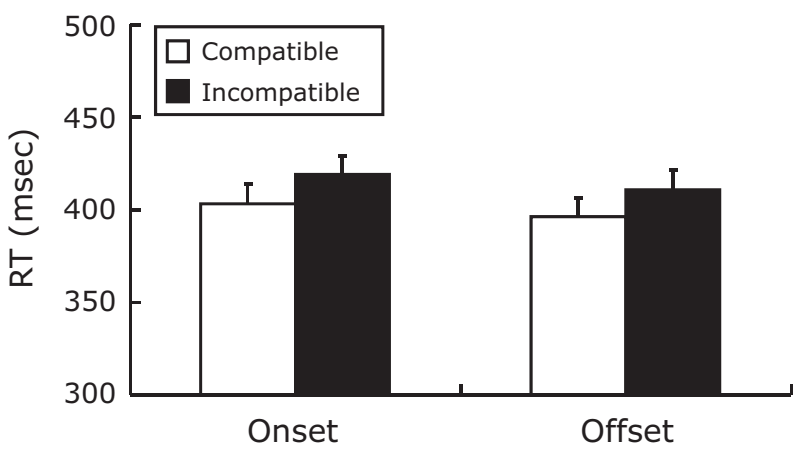

Figure 1. Mean reaction times (RTs, in milliseconds) in Experiment $1 \mathrm{~A}$ (upper panel) and $1 \mathrm{~B}$ (lower panel) as a function of condition and compatibility. Error bars represent standard errors of the means.

\section{Method}

Participants. Sixteen right-handed volunteers (7 females), 21-30 years of age $(M=24.9)$, took part in this experiment. None had participated in Experiment 1A. All of the participants reported that they had normal or corrected-to-normal vision. Three participants were replaced because of a high percentage of excluded trials (more than 10\%)

Apparatus, Stimuli, Task, and Procedure. These were identical to those in Experiment 1A, except for the following changes. The condition (onset vs. offset) was a within-participants factor and varied randomly within each block. A session consisted of five test blocks ( 80 trials per block), preceded by a practice block of 16 trials.

\section{Results}

Using the same criteria as in Experiment $1 \mathrm{~A}, 0.44 \%$ of the trials were excluded from the analyses. Mean RTs for correct responses and the percentages of errors were submitted to separate ANOVAs with condition (onset, offset) and compatibility (compatible, incompatible) as withinparticipants factors. The results are shown in Figure 1.

RT. The main effect of condition was significant $\left[F(1,15)=6.25, p<.05, \eta_{\mathrm{p}}^{2}=.294\right]$. Responses were faster for the offset condition $(M=404 \mathrm{msec})$ than for the onset condition $(M=411 \mathrm{msec})$. The main effect of compatibility was also significant $[F(1,15)=28.11, p<$ $\left..001, \eta_{\mathrm{p}}^{2}=.652\right]$, indicating the Simon effect. Overall, responses were faster for the compatible condition $(M=$
$400 \mathrm{msec})$ than for the incompatible condition $(M=$ $415 \mathrm{msec}$ ). The two-way interaction between condition and compatibility was not significant $(F<1)$. The Simon effect was comparable in size between the onset condition $(16 \mathrm{msec})$ and the offset condition $(15 \mathrm{msec})$.

Error rate. The main effects and the interaction were not significant $(p s>.19)$. The Simon effect was $-0.2 \%$ for the onset condition and $0.6 \%$ for the offset condition.

\section{Discussion}

The results of Experiment 1 are clear and consistent with the results of previous studies using stimulus offset (Simon et al., 1971; Wühr \& Kunde, 2006): Performance was better when the response and a visual change of an accessory stimulus were on the same side than when they were on opposite sides. This was true regardless of whether the change information involved the appearance or disappearance of an accessory stimulus. Moreover, the magnitude of the Simon effect was comparable in size for the onset and offset conditions. Although this conclusion is based on the null interaction, we obtained the same results regardless of whether the change condition (onset/offset) was a between-participants factor or varied within block as a within-participants factor. In this respect, the comparable accessory Simon effect for onset and offset conditions based on the transient visual change can be considered reliable. These results suggest that, in the visual modality, transient change exclusively determines the perceptual information that automatically activates the corresponding response, and that sustained stimulation from an ongoing stimulus does not contribute to this effect.

\section{EXPERIMENT 2}

\section{Auditory Accessory Stimulus}

Experiment 2 was identical to Experiment 1, except for the use of auditory accessory stimuli instead of visual accessory stimuli. Participants responded to the color of the central visual target with a right or left keypress. The accessory stimuli were presented to the right and left ears through headphones. Simon et al. (1971) showed, using the SRC task, that when either a right or a left accessory visual stimulus disappeared, the response associated with the corresponding side was faster than the response to the opposite side but that this was not the case for auditory accessory stimuli. For auditory stimuli, they found that responding was faster to the side where an auditory stimulus persisted. However, in their study, the identical tones were presented to the right and left ears. As a consequence, their participants heard a fused single tone perceptually originating from the center of their heads; when one of the tones disappeared, the resulting sound was perceived to be the onset of a new tone presented to the ear receiving persisting stimulation. In the present study, we used a pure tone and white noise as accessory stimuli, to avoid such a perception. This facilitates the correspondence between each sound and the presented ear. ${ }^{1}$ Again, the accessory stimulus change condition (onset, offset) was a between-participants factor in Experiment 2A and a within-participants factor in Experiment 2B. 


\section{Experiment 2A: Condition As a Between-Participants Factor}

\section{Method}

Participants. Forty volunteers ( 21 females; 6 left-handed), 20-33 years of age $(M=25.8)$, took part in this experiment. None had participated in the previous experiments. All of the participants reported that they had normal or corrected-to-normal vision and normal hearing. One participant was replaced because of a high percentage of excluded trials (more than 10\%).

Apparatus, Stimuli, Task, and Procedure. These were identical to those in Experiment 1, except for the following changes. Instead of visual accessory stimuli of right and left squares, we presented auditory accessory stimuli through headphones (AKG K271 Studio). Two different sounds functioned as accessory stimuli: (1) a $300-\mathrm{Hz}$ pure tone and (2) white noise. The intensity of each stimulus was approximately $76 \mathrm{~dB}$, and a linear amplitude ramp was applied for the first and the last $10 \mathrm{msec}$ of the duration. The white noise was generated with a bandwidth of $0-44.1 \mathrm{kHz}$; application of the Butterworth filter attenuated signal strength above $1.25 \mathrm{kHz}$ by $3 \mathrm{~dB}$ per octave.

On each of a series of trials, the sequence of visual stimulus presentation was exactly the same for both the onset and the offset conditions: A trial began with a 2,000-msec presentation of a white central fixation cross. The central visual fixation then changed color to yield a target color of either green or red, which remained for 2,000 msec. After that, the display went blank, and the intertrial interval lasted 1,000 msec. As in Experiment 1, the task required pressing either the right or the left key, according to the color of the central cross.

Accessory auditory stimuli were task irrelevant. For the onset condition, each trial started with the presentation of a central white fixation cross for $2,000 \mathrm{msec}$. Simultaneously with the presentation of a color change of the central cross (the target), either a pure tone or a white noise was monaurally presented to the right or the left ear through headphones for $2,000 \mathrm{msec}$. The sound ceased simultaneously with the offset of the colored central cross. For the offset condition, each trial started with the presentation of a pure tone and a white noise for $2,000 \mathrm{msec}$, one to the right ear and the other to the left ear (randomly determined), in addition to the presentation of the central white fixation cross. Simultaneously with the color change of the central cross (target), one of the two sounds was terminated. The remaining sound lasted for another $2,000 \mathrm{msec}$ and ceased simultaneously with the offset of the colored central cross.

\section{Results}

Using the same criteria as in Experiment 1A, 0.42\% of the trials were excluded from the analyses. Mean RTs for correct responses and the percentages of errors were submitted to separate ANOVAs with condition (onset, offset) as a between-participants factor and the type of the change sound (tone, noise) and compatibility (compatible, incompatible) as within-participants factors. Results are shown in Figure 2.

RT. The main effect of compatibility was significant $\left[F(1,38)=35.31, p<.001, \eta_{\mathrm{p}}^{2}=.482\right]$, indicating an overall Simon effect of $9 \mathrm{msec}$ (380 vs. $389 \mathrm{msec}$ for compatible vs. incompatible, respectively). The two-way interaction between compatibility and condition was significant $\left[F(1,38)=79.87, p<.001, \eta_{\mathrm{p}}^{2}=.678\right]$. The Simon effect was obtained for the sound onset condition ( $23 \mathrm{msec}, p<.001$ ) but not for the sound offset condition. In fact, a small reverse Simon effect $(-5 \mathrm{msec}, p<$ .05 ) was obtained for the offset condition; in other words, responses were faster when the response and the termina-

\section{Experiment 2A}

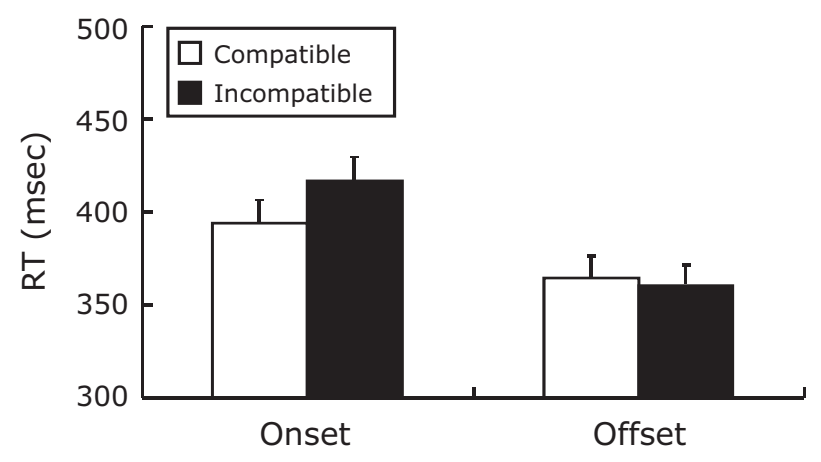

Experiment 2B

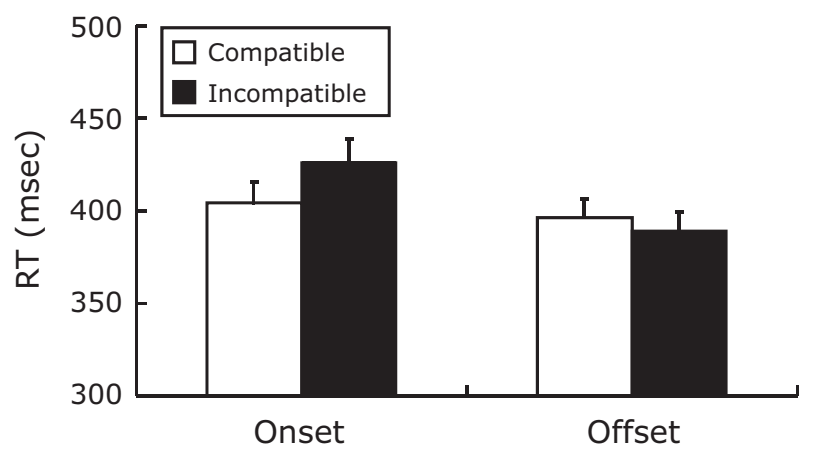

Figure 2. Mean reaction times (RTs, in milliseconds) in Experiment $2 \mathrm{~A}$ (upper panel) and $2 \mathrm{~B}$ (lower panel) as a function of condition and compatibility. Error bars represent standard errors of the means.

tion of a sound were on opposite sides than when they were on the same side. The main effect of condition was also significant $\left[F(1,38)=5.99, p<.05, \eta_{\mathrm{p}}^{2}=.136\right]$. Responses were slower for the onset condition $(M=$ $406 \mathrm{msec})$ than for the offset condition $(M=363 \mathrm{msec})$. Although responses tended to be faster when the changed sound was noise $(M=383 \mathrm{msec})$ than when it was a tone $(M=386 \mathrm{msec})$, the main effect of sound type missed significance $\left[F(1,38)=3.77, p=.060, \eta_{\mathrm{p}}^{2}=.090\right]$. Other interactions did not reach significance $\left(F_{\mathrm{S}}<1\right)$.

Error rate. Although the main effect of compatibility was not significant $(F<1)$, the two-way interaction between compatibility and condition was significant $\left[F(1,38)=9.70, p<.005, \eta_{\mathrm{p}}^{2}=.203\right]$. The Simon effect was obtained for the onset condition $(1.1 \%, p<.005)$ but not for the offset condition $(-0.6 \%$, n.s.). The main effect of condition was also significant $[F(1,38)=5.22, p<$ $\left..05, \eta_{\mathrm{p}}^{2}=.121\right]$. Participants responded more accurately for the onset condition (1.4\%) than for the offset condition (2.7\%). The two-way interaction between sound type and compatibility was significant $[F(1,38)=4.62, p<.05$, $\left.\eta_{\mathrm{p}}^{2}=.108\right]$, indicating a larger Simon effect for the tone change $(0.7 \%)$ than for the noise change $(-0.3 \%)$. The other main effect and interactions regarding sound type were not significant $(p s>.087)$. 


\section{Experiment 2B: Condition As a Within-Participants Factor}

\section{Method}

Participants. Twenty volunteers (6 females; 1 left-handed), 20-38 years of age $(M=29.4)$, took part in this experiment. None had participated in the previous experiments. All participants reported normal or corrected-to-normal vision and normal hearing. One participant was replaced because of a high percentage of the excluded trials (more than 10\%).

Apparatus, Stimuli, Task, and Procedure. These were identical to those in Experiment 2A, except for the following changes. The condition (onset vs. offset) was a within-participants factor and varied randomly within each block. A session consisted of five test blocks ( 80 trials per block), preceded by a practice block of 16 trials.

\section{Results}

Using the same criteria as in Experiment 1A, $0.63 \%$ of the trials were excluded from the analyses. Mean RTs for correct responses and the percentages of errors were submitted to separate ANOVAs with condition (onset, offset), type of the changed sound (tone, noise), and compatibility (compatible, incompatible) as within-participants factors. Results are shown in Figure 2.

RT. The main effect of compatibility was significant $\left[F(1,19)=14.45, p<.005, \eta_{\mathrm{p}}^{2}=.432\right]$, indicating an overall Simon effect of $8 \mathrm{msec}$. The two-way interaction between compatibility and condition was significant $\left[F(1,19)=73.16, p<.001, \eta_{\mathrm{p}}^{2}=.794\right]$. The Simon effect was obtained for the sound onset condition $(22 \mathrm{msec}$, $p<.001)$, whereas a significant reverse Simon effect $(-7 \mathrm{msec}, p<.05)$ was obtained for the offset condition; in other words, responses were faster when the response and the termination of a sound were on opposite sides than when they were on the same side. The main effect of condition was significant $[F(1,19)=37.37, p<.001$, $\left.\eta_{\mathrm{p}}^{2}=.663\right]$. Responses were slower for the onset condition $(M=415 \mathrm{msec})$ than for the offset condition $(M=$ $392 \mathrm{msec}$ ). Concerning the type of the changed sound, neither the main effect of sound type $[F(1,19)=3.22$, $\left.p=.089, \eta_{\mathrm{p}}^{2}=.145\right]$ nor the two-way interaction between compatibility and sound type $(F<1)$ was significant. However, both the two-way interaction between condition and sound type $\left[F(1,19)=5.32, p<.05, \eta_{\mathrm{p}}^{2}=.219\right]$ and the three-way interaction between condition, compatibility, and sound type $\left[F(1,19)=4.93, p<.05, \eta_{\mathrm{p}}^{2}=\right.$ .206] were significant. Separate ANOVAs for each condition revealed that, in the onset condition, responses were faster $\left[F(1,19)=10.04, p<.01, \eta_{\mathrm{p}}^{2}=.346\right]$ and the size of the Simon effect tended to be larger $[F(1,19)=3.67$, $\left.p=.071, \eta_{\mathrm{p}}^{2}=.162\right]$ when noise was presented $(M=$ $410 \mathrm{msec}$; Simon effect $=26 \mathrm{msec}$ ) than when a tone was presented $(M=419 \mathrm{msec}$; Simon effect $=18 \mathrm{msec})$. In contrast, in the offset condition, the type of the terminated sound did not alter the results $(p \mathrm{~s}>.15)$.

Error rate. Although the main effect of compatibility was not significant $\left[F(1,19)=3.05, p=.097, \eta_{\mathrm{p}}^{2}=.138\right]$, the two-way interaction between compatibility and condition was significant $\left[F(1,19)=25.75, p<.001, \eta_{\mathrm{p}}^{2}=\right.$ $.575]$. The Simon effect was obtained for the onset condition $(2.2 \%, p<.001)$, whereas a reverse Simon effect was obtained for the offset condition $(-1.0 \%, p<.05)$. The main effect of sound type was significant $[F(1,19)=$ $\left.7.75, p<.05, \eta_{\mathrm{p}}^{2}=.290\right]$. Participants responded more accurately for the tone change $(1.9 \%)$ than for the noise change $(2.6 \%)$. The other main effects and interactions were not significant $(F \mathrm{~s}<1)$.

\section{Discussion}

For the onset condition, we successfully replicated the auditory accessory Simon effect (e.g., Proctor et al., 2005; Simon \& Craft, 1970; Simon \& Pouraghabagher, 1978): Responses on the side associated with the onset of a sound (tone or noise) were faster and more accurate than responses on the side opposite to the onset of a sound. In contrast, for the offset condition, responses on the side of the ongoing sound were slightly faster than responses on the side associated with a sound's termination. Although numerically small, a reverse accessory Simon effect was obtained for both Experiments 2A and 2B. Therefore, we conclude that the reverse accessory Simon effect for the sound offset condition is reliable. The findings with accessory sound stimulus change suggest that sustained stimulation from an ongoing auditory stimulus is important in the automatic activation of the spatially corresponding response, but that transient change information also contributes, in the auditory modality.

Overall, RTs were shorter (significantly so for both Experiments $2 \mathrm{~A}$ and $2 \mathrm{~B}$ ), whereas error rate tended to be higher (significant for Experiment 2A but not for Experiment 2B) for the offset condition than for the onset condition, indicating a speed-accuracy trade-off between the onset and offset conditions. Despite this trade-off, the effect of a sound's termination on the right and left responses was similar in RTs and in error rates. The reverse Simon effect was observed in both measures for the offset condition. (Although the reverse Simon effect was not significant in error rates for Experiment 2A, the numerical trend toward the reverse Simon effect was observed even in this condition.) The effects of changed sound type on performance were not consistent across experiments. Most importantly, the sound type did not alter the basic pattern of a positive Simon effect for the onset condition and a smaller negative Simon effect for the offset condition.

\section{GENERAL DISCUSSION}

The present study investigated a perceptual property that automatically facilitates a feature-sharing action in visual and auditory modalities. Using disappearance of an accessory stimulus, we dissociated the effects of transient changes from potential effects due to sustained input associated with the mere presence of a stimulus object, with the aim of isolating causal sources of the Simon effect.

In Experiment 1, the results revealed effects of both types of transient change, onsets and offsets, associated with an accessory visual stimulus. Transient change influenced accuracy and timing of responses to a visual target despite sustained stimulation of the accessory stimulus. In Experiment 2, the results indicated that both the sustained input from an accessory auditory stimulus and a transient 
change in auditory stimulation contribute to accuracy and RTs to a visual target and that the contribution of the former is more prominent than the latter. In other words, in the auditory modality, participants revealed a strong tendency to be influenced by ongoing (sustained) stimulus information rather than by stimulus change. These findings suggest that the contribution of sustained stimulation is more prominent in this modality than in vision.

In both experiments, the results are based on events related to task-irrelevant accessory stimuli within visual/ auditory fields and cannot be attributed to a memory-based explanation (Hommel, 2002; Zhang \& Johnson, 2004; see the introduction). These results were robust and consistent across experimental designs; similar outcomes emerged in designs in which each participant experienced only one kind of change - that is, the onset or offset condition, over trials - and in which participants experienced both onset and offset conditions mixed within a trial block. Thus, we conclude that the results of the present study reflect a general, automatic effect of perception on action that does not depend on participants' specific intentions or strategies to perform the task.

One purpose of the present study was to test whether visual disappearance induces automatic activation of a response on the corresponding side, even when the task engenders no potential demand for memory retrieval of a stimulus that has disappeared. Experiment 1 successfully replicated the Simon effect induced by visual stimulus offset (Wühr \& Kunde, 2006) while excluding the potential confounding factor of a memory-based Simon effect (Hommel, 2002; Zhang \& Johnson, 2004). The Simon effect observed in Experiment 1 was comparable in size for both the accessory stimulus onset and offset conditions. Such findings confirm that transient change information elicits response activation on the corresponding side in visual information processing.

Another purpose of the present research was to test whether the effect of stimulus disappearance on response selection is comparable across presentation modalities of an accessory stimulus. Using visual stimuli in Experiment 1 and auditory stimuli in Experiment 2, we found that effects due to disappearance varied with modality. Although auditory accessory stimuli yielded results comparable to those found with visual accessory stimuli for the onset condition, in the offset condition, auditory offsets produced slightly faster responses on the opposite side, which is contrary to visual offsets, produce faster responses on the corresponding side. Hereafter, we discuss explanations for these modality differences.

Aside from the modality difference itself, a potentially relevant design difference accompanied the modality manipulation in this study. In both Experiments 1 and 2, the target was a visual stimulus; but the design of Experiment 1 was unimodal, in that both relevant (target) and irrelevant (accessory) stimuli were visual, whereas the design of Experiment 2 was cross-modal, in that targets were visual and accessory stimuli were auditory. This raises the possibility that the explanation of differences in the two experiments involves constraints related to unimodal versus cross-modal designs. One explanatory approach builds on the assumption that the unimodal design of Experiment 1 involves shared, modality-specific resources, whereas the cross-modal design of Experiment 2 does not.

In considering this approach, we note that the unimodal assumption implies that in the offset condition of Experiment 1, the visual target and the remaining visual accessory stimuli should compete for modality-specific (visual) resources (see Desimone \& Duncan, 1995). Building upon this, such competition would highlight contrasts between target and accessory stimuli. In turn, a contrast may allow the sustaining accessory stimulus to serve as a potent reference object for spatial coding of the central target. According to the referential coding account of the Simon effect (Hommel, 1993), the target stimulus is coded with regard to reference object(s), such as fixation and/or other salient objects. Therefore, in principle, any accessory stimulus that occurs together on the screen with the target should function as a reference object. Consequently, this explanation implies that the central target is coded relative to a visible accessory stimulus location. For example, when the accessory stimulus remained on the screen on the right side of the central target, the target would be coded as left, relative to the accessory stimulus. This coding would facilitate the left response, which is on the side of the accessory stimulus offset.

A different picture emerges in the offset condition of the cross-modal design of Experiment 2. In this case, the visual target and the remaining auditory accessory stimuli should not compete with each other for attentional resources (Duncan, Martens, \& Ward, 1997). Instead, the visual target and auditory accessory stimulus may be cross-modally integrated to form a single perceptual event (see de Gelder \& Bertelson, 2003). This unified percept could result in an assignment of spatial property that corresponds to the side of the sustained (accessory) auditory stimulus to the central visual target. In this case, the imbued spatial property could accelerate the response on the side corresponding to the simultaneously ongoing sound.

This account of unimodal and cross-modal influences on spatial coding may explain the differential influence of offsets as a function of modality, but it cannot address performance in the visual onset condition. That is, the above account fails because it predicts faster responses to targets when visual onsets of accessory stimuli happen on the opposite side from a response (i.e., incompatible onsets in Experiment 1). This is because this approach assumes that the visual accessory stimulus that has appeared will function as the reference object, relative to which the target is spatially coded. Contrary to this prediction, the results showed faster responses for compatible onsets than for incompatible onsets in Experiment 1.

Moreover, others have reported results suggesting that the strong influence of ongoing auditory stimulation (vs. ongoing visual stimulation) is not restricted to crossmodal situations. For instance, using the SRC task, Simon et al. (1971) found that responses were faster when the disappearance of the visual stimulus was compatible with the spatial location of a response, whereas the opposite was true with auditory stimuli. Effects of a task-irrelevant noise stimulus in the unimodal Simon effect also differ be- 
tween visual and auditory modalities. With a visual Simon task, a visual noise stimulus in the location opposite to the target location did not reduce the Simon effect, and in some cases, the Simon effect increased by this manipulation (e.g., Proctor \& Lu, 1994). Simon, Craft, and Small (1970) investigated the effect of an auditory noise stimulus presented to the ear opposite the ear receiving a target tone. Participants pressed a right or left key in response to the frequency of a target tone presented to either the right or the left ear. When the target tone was accompanied by a noise presented to the opposite ear, unlike in the visual case, the Simon effect was reduced, eliminated, or even reversed as a function of the noise intensity at the opposite ear. When the target tone was accompanied by a noise in the same ear, the Simon effect was enhanced. Thus, with respect to the effect of perception on action, the auditory modality appears to elicit an automatic response to input information, based on the source of auditory stimulation, even in the unimodal situation. Cross-modality does not seem to be sufficient to fully explain the modality differences in the present study.

An alternative explanation of the present findings focuses on the role of attention with stimuli of different modalities. The close relationship between spatial attention and the Simon effect has been discussed in literature (e.g., Lleras, Moore, \& Mordkoff, 2004; Nicoletti \& Umiltà, 1989; Notebaert, Soetens, \& Melis, 2001; Rubichi, Nicoletti, Iani, \& Umiltà, 1997; Stoffer, 1991; Treccani et al., 2006). Stimulus appearance - that is, onsets - can capture attention, and the sudden appearance of an object plays a prominent role in the Simon effect. Eyes (see, e.g., Driver et al., 1999; Friesen \& Kingstone, 1998), arrows (see, e.g., Eimer, 1997; Hommel, Pratt, Colzato, \& Godijn, 2001), spatial words (see, e.g., Hommel et al., 2001), and numbers (see, e.g., Fischer, Castel, Dodd, \& Pratt, 2003) are known to cause an automatic shift of attention. They also cause automatic activation of horizontally arranged responses, inducing effects similar to the Simon effect (e.g., Ansorge, 2003; Baldo, Shimamura, \& Prinzmetal, 1998; Dehaene, Bossini, \& Giraux, 1993; Eimer, 1995; Lu \& Proctor, 2001; Masaki, Takasawa, \& Yamazaki, 2000; Ricciardelli, Bonfiglioli, Iani, Rubichi, \& Nicoletti, 2007; Zorzi, Mapelli, Rusconi, \& Umiltà, 2003). Visual offset is also known to attract attention (e.g., Chastain \& Cheal, 2001; Theeuwes, 1991). Experiment 1 of the present study revealed that a visual offset automatically activates a spatially congruent response. Thus, the present study supplies further support for the notion of a close relationship between spatial attention and the Simon effect. In terms of this attention shift account, a transient change of a visual accessory stimulus attracts attention to its spatial location, thereby automatically activating a spatially congruent response.

Applying the attention shift hypothesis to auditory accessory stimuli rests on the assumption that information extracted from these stimuli, and/or cognitive weighting of this information, is less potent for auditory offsets than for auditory onsets (see Phillips, Hall, \& Boehnke, 2002). Therefore, termination of an auditory stimulus in synchrony with the onset of a visual target in Experiment 2 is less likely to elicit a strong, automatic shift of attention to the accessory stimulus offset location than is a synchronized auditory onset. Lacking an attention shift to the side of the offset, an auditory offset should also exert less influence than do auditory onsets on response selection. This account explains the differential contributions of onsets and offsets in the Simon effect due to modality.

In the visual modality, transient change determined response activation without contribution of sustained stimulation. In the auditory modality, sustained stimulation determined the response activation, with a relatively small contribution of transient change. This modality difference may reflect the differential nature of spatial coding in the two modalities. The importance of transient change in vision indicates that visual presentations promote spatial coding of events in the environment, independently of ongoing stimulation input to the perceptual organ. On the other hand, auditory spatial information processing may rely more on physical stimulation input to the perceptual organ - that is, the left/right ear. The importance of physically related coding in auditory information processing was also indicated in the response action coding when hands were crossed. The coding of response action on the basis of the spatial location in the environment (i.e., left/ right coding on the basis of the response-key location) determined the left/right response representation to the visual stimuli, whereas the effector coding of the response action (i.e., the left/right coding on the basis of the effector identity of the responding hand) also contributed to the left/right response representation to the auditory stimuli (Roswarski \& Proctor, 2003; Wascher, Schatz, Kuder, \& Verleger, 2001). The spatial coding difference between visual and auditory modalities would determine the contribution of transient change and sustained input in perceptual and cognitive information processing in each modality. These differences may be responsible for observed differences in automatic response activation, as well as the differential role of spatial attention in the two modalities.

Finally, another line of evidence also suggests the difference between the Simon effects with spatial coding based on the environmental space and on the stimulation to the perceptual organ. Valle-Inclán, Hackley, and de Labra (2003) reported a small Simon effect on the basis of the stimulation of the visual perceptual organ - that is, the left/right eye. Distinct mechanisms underlying the standard Simon effect and this monocular Simon effect were recently reported by Schankin, Valle-Inclán, and Hackley (2010). The contribution of the effect of correspondence between responses and the perceiver's stimulated perceptual organ would be large in the auditory Simon effect.

\section{AUTHOR NOTE}

This study was supported by a grant from the Research Fellowships of the Japan Society for the Promotion of Science for Young Scientists to A.N., and by a Grant-in-Aid for Scientific Research from the Japan Society for the Promotion of Science, awarded to K.Y. A.N. is now at Sophia University as a JSPS Research Fellow (e-mail: a-nishimura@ sophia.ac.jp). We thank Naoko Sato for her help generating the auditory stimuli, and Simon Grondin, Bernhard Hommel, and two anonymous reviewers for their helpful and constructive comments. Correspondence concerning this article should be addressed to K. Yokosawa, Department 
of Psychology, Graduate School of Humanities and Sociology, University of Tokyo, 7-3-1 Hongo, Bunkyo-ku, Tokyo 113-0033, Japan (e-mail: yokosawa@L.u-tokyo.ac.jp).

\section{REFERENCES}

Ansorge, U. (2003). Spatial Simon effects and compatibility effects induced by observed gaze direction. Visual Cognition, 10, 363-383. doi:10.1080/13506280244000122

Baldo, J. V., Shimamura, A. P., \& Prinzmetal, W. (1998). Mapping symbols to response modalities: Interference effects on Stroop-like tasks. Perception \& Psychophysics, 60, 427-437.

Chastain, G., \& Cheal, M. (2001). Attentional capture with various distractor and target types. Perception \& Psychophysics, 63, 979-990.

DE GELDER, B., \& BerTelson, P. (2003). Multisensory integration, perception and ecological validity. Trends in Cognitive Sciences, 7, 460467. doi:10.1016/j.tics.2003.08.014

Dehaene, S., Bossini, S., \& Giraux, P. (1993). The mental representation of parity and number magnitude. Journal of Experimental Psychology: General, 122, 371-396. doi:10.1037/0096-3445.122.3.371

Desimone, R., \& Duncan, J. (1995). Neural mechanisms of selective visual attention. Annual Review of Neuroscience, 18, 193-222. doi:10.1146/annurev.ne.18.030195.001205

Deubel, H., Schneider, W. X., \& Paprotta, I. (1998). Selective dorsal and ventral processing: Evidence for a common attentional mechanism in reaching and perception. Visual Cognition, 5, 81-107. doi:10.1080/713756776

Driver, J., Davis, G., Ricciardelli, P., Kidd, P., Maxwell, E., \& BARON-Cohen, S. (1999). Gaze perception triggers reflexive visuospatial orienting. Visual Cognition, 6, 509-540. doi:10.1080/ 135062899394920

Duncan, J., Martens, S., \& Ward, R. (1997). Restricted attentional capacity within but not between sensory modalities. Nature, 387, 808810. doi: $10.1038 / 42947$

EIMER, M. (1995). Stimulus-response compatibility and automatic response activation: Evidence from psychophysiological studies. Journal of Experimental Psychology: Human Perception \& Performance, 21, 837-854. doi:10.1037/0096-1523.21.4.837

EIMER, M. (1997). Uninformative symbolic cues may bias visual-spatial attention: Behavioral and electrophysiological evidence. Biological Psychology, 46, 67-71. doi:10.1016/S0301-0511(97)05254-X

Fischer, M. H., Castel, A. D., Dodd, M. D., \& Pratt, J. (2003). Perceiving numbers causes spatial shifts of attention. Nature Neuroscience, 6, 555-556. doi:10.1038/nn1066

Friesen, C. K., \& Kingstone, A. (1998). The eyes have it! Reflexive orienting is triggered by nonpredictive gaze. Psychonomic Bulletin \& Review, 5, 490-495.

Hommel, B. (1993). The role of attention for the Simon effect. Psychological Research, 55, 208-222. doi:10.1007/BF00419608

Hommel, B. (2002). Responding to object files: Automatic integration of spatial information revealed by stimulus-response compatibility effects. Quarterly Journal of Experimental Psychology, 55A, 567-580. doi:10.1080/02724980143000361

Hommel, B., Pratt, J., Colzato, L., \& Godijn, R. (2001). Symbolic control of visual attention. Psychological Science, 12, 360-365. doi:10.1111/1467-9280.00367

Kornblum, S., HasbroucQ, T., \& Osman, A. (1990). Dimensional overlap: Cognitive basis for stimulus-response compatibility - A model and taxonomy. Psychological Review, 97, 253-270. doi:10.1037/0033 $-295 X .97 .2 .253$

LidJi, P., Kolinsky, R., Lochy, A., \& Morais, J. (2007). Spatial associations for musical stimuli: A piano in the head? Journal of Experimental Psychology: Human Perception \& Performance, 33, 1189-1207. doi:10.1037/0096-1523.33.5.1189

Lleras, A., Moore, C. M., \& Mordkoff, J. T. (2004). Looking for the source of the Simon effect: Evidence for multiple codes. American Journal of Psychology, 117, 531-542. doi:10.2307/4148990

Lu, C.-H., \& Proctor, R. W. (1995). The influence of irrelevant location information on performance: A review of the Simon and spatial Stroop effects. Psychonomic Bulletin \& Review, 2, 174-207.

LU, C.-H., \& Proctor, R. W. (2001). Influence of irrelevant information on human performance: Effects of S-R association strength and relative timing. Quarterly Journal of Experimental Psychology, 54A, 95-136. doi:10.1080/02724980042000048

Masaki, H., TaKasawa, N., \& YamazaKi, K. (2000). An electrophysiological study of the locus of the interference effect in a stimulusresponse compatibility paradigm. Psychophysiology, 37, 464-472. doi: $10.1017 /$ S0048577200981976

Moore, C. M., Lleras, A., Grosjean, M., \& Marrara, M. T. (2004). Using inattentional blindness as an operational definition of unattended: The case of a response-end effect. Visual Cognition, 11, 705719. doi:10.1080/13506280344000482

Nicoletti, R., \& Umiltà, C. (1989). Splitting visual space with attention. Journal of Experimental Psychology: Human Perception \& Performance, 15, 164-169. doi:10.1037/0096-1523.15.1.164

Nishimura, A., \& Yokosawa, K. (2009). Effects of laterality and pitch height of an auditory accessory stimulus on horizontal response selection: The Simon effect and the SMARC effect. Psychonomic Bulletin \& Review, 16, 666-670. doi:10.3758/PBR.16.4.666

Notebaert, W., Soetens, E., \& Melis, A. (2001). Sequential analysis of a Simon task: Evidence for an attention-shift account. Psychological Research, 65, 170-184. doi:10.1007/s004260000054

Phillips, D. P., Hall, S. E., \& BoennKe, S. E. (2002). Central auditory onset responses, and temporal asymmetries in auditory perception. Hearing Research, 167, 192-205.

PosNer, M. I. (1980). Orienting of attention. Quarterly Journal of Experimental Psychology, 32, 3-25. doi:10.1080/00335558008248231

Proctor, R. W., \& LU, C.-H. (1994). Referential coding and attentionshifting accounts of the Simon effect. Psychological Research, 56, 185-195. doi:10.1007/BF00419706

Proctor, R. W., Pick, D. F., Vu, K.-P. L., \& Anderson, R. E. (2005). The enhanced Simon effect for older adults is reduced when the irrelevant location information is conveyed by an accessory stimulus. Acta Psychologica, 119, 21-40. doi:10.1016/j.actpsy.2004.10.014

Proctor, R. W., \& Vu, K.-P. L. (2006). Stimulus-response compatibility principles: Data, theory, and application. Boca Raton, FL: CRC Press.

Ricciardelli, P., Bonfiglioli, C., Iani, C., Rubichi, S., \& NicoLETTI, R. (2007). Spatial coding and central patterns: Is there something special about the eyes? Canadian Journal of Experimental Psychology, 61, 79-90. doi:10.1037/cep2007_2_79

Roswarski, T. E., \& Proctor, R. W. (2003). The role of instructions, practice, and stimulus-hand correspondence on the Simon effect. Psychological Research, 67, 43-55.

Rubichi, S., Nicoletti, R., IANi, C., \& Umiltà, C. (1997). The Simon effect occurs relative to the direction of an attention shift. Journal of Experimental Psychology: Human Perception \& Performance, 23, 1353-1364. doi:10.1037/0096-1523.23.5.1353

Rusconi, E., Kwan, B., Giordano, B. L., Umiltà, C., \& ButterWORTH, B. (2006). Spatial representation of pitch height: The SMARC effect. Cognition, 99, 113-129. doi:10.1016/j.cognition.2005.01.004

Schankin, A., Valle-Inclán, F., \& Hackley, S. A. (2010). Compatibility between stimulated eye, target location and response location. Psychological Research, 74, 291-301. doi:10.1007/s00426-009 $-0247-\mathrm{x}$

SChröter, H., Ulrich, R., \& Miller, J. (2007). Effects of redundant auditory stimuli on reaction time. Psychonomic Bulletin \& Review, 14, 39-44.

Simon, J. R. (1990). The effects of an irrelevant directional cue on human information processing. In R. W. Proctor \& T. G. Reeve (Eds.), Stimulus-response compatibility: An integrated perspective (pp. 3186). Amsterdam: North-Holland.

Simon, J. R., \& CRAFt, J. L. (1970). Effects of an irrelevant auditory stimulus on visual choice reaction time. Journal of Experimental Psychology, 86, 272-274. doi:10.1037/h0029961

Simon, J. R., Craft, J. L., \& Small, A. M., JR. (1970). Manipulating the strength of a stereotype: Interference effects in an auditory information-processing task. Journal of Experimental Psychology, 86, 63-68. doi:10.1037/h0029944

Simon, J. R., Craft, J. L., \& Webster, J. B. (1971). Reaction time to onset and offset of lights and tones: Reactions toward the changed element in a two-element display. Journal of Experimental Psychology, 89, 197-202. doi: $10.1037 / \mathrm{h} 0031167$

Simon, J. R., \& Pouraghabagher, R. (1978). The effect of aging on 
the stages of processing in a choice reaction time task. Journal of Gerontology, 33, 553-561.

Simon, J. R., \& Small, A. M., JR. (1969). Processing auditory information: Interference from an irrelevant cue. Journal of Applied Psychology, 53, 433-435. doi:10.1037/h0028034

Stoffer, T. H. (1991). Attentional focussing and spatial stimulusresponse compatibility. Psychological Research, 53, 127-135. doi:10.1007/BF01371820

TheEuwes, J. (1991). Exogenous and endogenous control of attention: The effect of visual onsets and offsets. Perception \& Psychophysics, 49, 83-90.

Treccani, B., Umiltà, C., \& Tagliabue, M. (2006). Simon effect with and without awareness of the accessory stimulus. Journal of Experimental Psychology: Human Perception \& Performance, 32, 268-286. doi: $10.1037 / 0096-1523.32 .2 .268$

Umiltà, C., \& Nicoletti, R. (1990). Spatial stimulus-response compatibility. In R. W. Proctor \& T. G. Reeve (Eds.), Stimulus-response compatibility: An integrated perspective (pp. 89-116). Amsterdam: North-Holland.

Valle-Inclán, F., Hackley, S. A., \& DE Labra, C. (2003). Stimulusresponse compatibility between stimulated eye and response location: Implications for attentional accounts of the Simon effect. Psychological Research, 67, 240-243. doi:10.1007/s00426-003-0131-z

Wascher, E., Schatz, U., Kuder, T., \& Verleger, R. (2001). Validity and boundary conditions of automatic response activation in the Simon task. Journal of Experimental Psychology: Human Perception \& Performance, 27, 731-751. doi:10.1037/0096-1523.27.3.731
WüHR, P., \& KundE, W. (2006). Spatial correspondence between onsets and offsets of stimuli and responses. European Journal of Cognitive Psychology, 18, 359-377. doi:10.1080/09541440500334417

Zhang, J. X., \& Johnson, M. K. (2004). A memory-based, Simonlike, spatial congruence effect: Evidence for persisting spatial codes. Quarterly Journal of Experimental Psychology, 57A, 419-436. doi:10.1080/02724980343000404

Zorzi, M., Mapelli, D., Rusconi, E., \& Umiltà, C. (2003). Automatic spatial coding of perceived gaze direction is revealed by the Simon effect. Psychonomic Bulletin \& Review, 10, 423-429.

\section{NOTE}

1. We used a pure tone and noise instead of two pure tones with different frequencies as auditory stimuli because two pure tones tended to be fused into a single percept and, as a result, localization of each sound would be difficult (Schröter, Ulrich, \& Miller, 2007). Moreover, two tones with different frequencies are known to have associations with right and left responses on the basis of their pitch height (Lidji, Kolinsky, Lochy, \& Morais, 2007; Nishimura \& Yokosawa, 2009; Rusconi, Kwan, Giordano, Umiltà, \& Butterworth, 2006). Use of two tones with different pitch height would make the situation complicated.

(Manuscript received July 15, 2008; revision accepted for publication May 11, 2010.) 